12

\title{
Безэталонный анализ твердых веществ масс-спектрометром с индуктивно связанной плазмой
}

\author{
(C) Т.К. Нурубейли, З.К. Нурубейли, К.З. Нуриев, К.Б. Гурбанов \\ Институт Физики НАН Азербайджана, \\ Az-1143 Баку, Азербайджан \\ e-mail: kamilnuri@rambler.ru
}

(Поступило в Редакцию 20 октября 2015 г. В окончательной редакции 8 июня 2016 г.)

\begin{abstract}
Рассмотрена возможность безэталонного масс-спектрометрического анализа элементного состава твердых тел. Теоретически и экспериментально исследовано влияние каждого этапа разлета лазерной плазмы на формирование коэффициента относительной чувствительности элементов пробы. Показано, что основные вклады в формирование коэффициента относительной чувствительности внесены этапами ионообразования и детектирования. Предложено разделить процессы диссоциации и ионизации по времени и (или) по пространству. Для компенсации энергетического разброса ионов на выходе из анализатора предложена схема для выравнивания энергий ионов перед их детектированием.
\end{abstract}

DOI: 10.21883/JTF.2017.02.44138.1624

\section{Введение}

Масс-спектрометрия с плазменными источниками ионов (искровым, лазерным, тлеющим разрядом, вторичной ионной эмиссией (ВИЭ)) является одним из ведущих методов высокочувствительного анализа твердых веществ [1,2]. Эти методы в принципе позволяют регистрировать даже единичные ионы [3]. Однако измеренный состав ионов в большинстве случаев не соответствует истинному составу пробы. Это несоответствие отношений линий в масс-спектрах концентраций ионов в анализируемом образце обычно учитывают с помощью коэффициента относительной чувствительности Coefficient of Relative Sensitivity (CRS) определяемого элемента по отношению к внутреннему стандарту (Internal Standart, IS), т. е. элементу с известным составом и количеством [1-5], вводимому в состав образца во время пробоподготовки

$$
\mathrm{CRS}_{\mathrm{IS}}^{x}=\frac{J_{x}}{J_{\mathrm{IS}}} \frac{n_{\mathrm{IS}}}{n_{x}}
$$

где $I_{x}, J_{\mathrm{IS}}$ - измеренные аналитические сигналы определяемого элемента и внутреннего стандарта, $n_{x}, n_{\mathrm{IS}}-$ концентрации соответствующих элементов. При этом концентрации искомых элементов определялись относительно внутреннего стандарта.

Из-за большого разнообразия элементов в составе анализируемых образцов, а также из-за того, что на формирование CRS влияют почти все стадии массспектрометрического анализа, экспериментальное определение CRS является нереальной задачей [4,5]. Было решено CRS различных элементов определить теоретически, что привело к появлению большого числа эмпирических выражений [6], в основе которых лежали почти столько же механизмов образования плазмы, сколько и „работали“ только для данного выражения, и только при определенных условиях эксперимента.
Поэтому на практике часто пытаются осуществлять так называемый безэталонный анализ [7-10]. Он основан на предположении, что CRS для всех элементов равен единице. Такой подход приводит к систематической погрешности до 100\% [3,5]. Другая возможность основана либо на использовании стандартных образцов, адекватных анализируемым объектам по составу матрицы, либо на расчете средних значений CRS по результатам анализа множества образцов с различными матрицами. Обеспечиваемая при таком подходе относительная систематическая погрешность примерно такая же, что и в предположении, что CRS для всех элементов равняется единице.

Целью настоящей работы является попытка исследовать возможности проведения количественного массспектрометрического анализа твердых веществ с минимальной систематической погрешностью измерений без использования внутренних стандартов.

\section{Теоретическая часть}

Для решения проблемы более реальным представляется путь развития теории, которая позволила бы рассчитывать значения CRS априори, с учетом основных параметров определяемых элементов, свойства матрицы, а также, что очень важно, условий проведения эксперимента. При таком подходе CRS можно представить как интегральную характеристику, учитывающую дискриминацию атомов и ионов элементов примеси в источнике ионов, обусловленных диссоциацией $\mathrm{CRS}_{a}$, ионизацией $\mathrm{CRS}_{\text {ion }}$ и рекомбинационных процессов $\mathrm{CRS}_{\mathrm{rec}}$, протекающих в разлетающейся плазме, а также дискриминацию ионов по массам (энергиям) и (или) зарядам при разделении их в масс-анализаторе $\mathrm{CRS}_{\mathrm{an}}$ и детекторе $\mathrm{CRS}_{\text {det }}[4]$.

$$
\mathrm{CRS}_{i}=\mathrm{CRS}_{a} \cdot \mathrm{CRS}_{\mathrm{ion}} \cdot \mathrm{CRS}_{\mathrm{rec}} \cdot \mathrm{CRS}_{\mathrm{an}} \cdot \mathrm{CRS}_{\mathrm{det}} \cdot
$$


Выделение в CRS отдельных составляющих упрощает решение задачи, поскольку за каждый конкретный вид дискриминации отвечает отдельный физический процесс, поддающийся теоретическому описанию. Из анализа физических процессов на стадии ионизации следует, что при расчете $\mathrm{CRS}_{\text {ion }}$ необходимо учитывать следующее. Во-первых, на начальной стадии разлета энергия, накопленная плазмой, достаточна для поддержания ионизационных процессов. Во-вторых, при определении степени ионизации плазмы необходимо учитывать тот факт, что формула Саха справедлива для двукомпонентной плазы, и в случае присутствия в плазме ионов разных масс необходима ее коррекция (уравление СахаЭггерта). В-третьих, при рассмотрении лазерной плазмы (равно как и искровой и ВИЭ) можно говорить только о локальном ее равновесии $[8,9,11,12]$.

Отметим, что дискриминацию ионов в масс-анализаторе и детектирующем устройстве можно учесть путем тщательной градуировки ионно-оптического тракта, о котором пойдет речь ниже. Учет же дискриминации ионов в плазме в принципе более сложен и требует рассмотрения физики протекающих процессов.

В ряде работ [3,13-15] предложено применить для этой цели различные модели, основанные на предположении существования в плазме того или иного типа равновесий [14], но это не дало результатов. В большинстве работ показано, что как в высокотемпературной плазме (искровой и лазерной) $[15,16]$, так и в низкотемпературной плазме (индуктивно связанной плазме и тлеющего разряда) равновесия не существует. Описание же неравновесных процессов очень сложно как с физической, так и математической точек зрения. Поэтому в [17] нами подробно рассматривалось влияние рекомбинации в разлетающейся лазерной плазме на распределение ионов по разрядностям. При этом, отвлекаясь от обсуждения процессов, приводящих к образованию плазменного сгустка, принимается, что плазма образовалась в результате диссоциации и ионизации атомов вещества, в котором установилось локальное (пространственное и временное) термодинамическое равновесие, а исходные температуры ионов и электронов равны $T_{i}$ и $T_{e}$ соответственно. В такой плазме оценивается роль рекомбинации в формировании ионного состава разлетающейся лазерной плазмы.

Расчеты показывают, что если длина рекомбинации ионов с зарядом $z$ меньше начального радиуса плазмы $\left(l_{\mathrm{rec}}^{z} \ll R_{0}\right)$, то ионы данной зарядности практически полностью рекомбинируют за время $t_{r}=R_{0} \sqrt{M T_{e}}$ (где $M$ - масса иона) и, начиная с $t=4 t_{r}$, все неупругие процессы полностью прекращаются, хотя температура плазменного сгустка продолжает падать, причем температура электронов уменьшается медленнее, чем температура ионов (рис. 1). Это объясняется тем, что в процессе рекомбинации энергия ионов перекачивается сначала в электронный газ, а потом передается ионам и в конечном счете превращается в энергию направленного движения ионов. В результате охлаждения плазмы

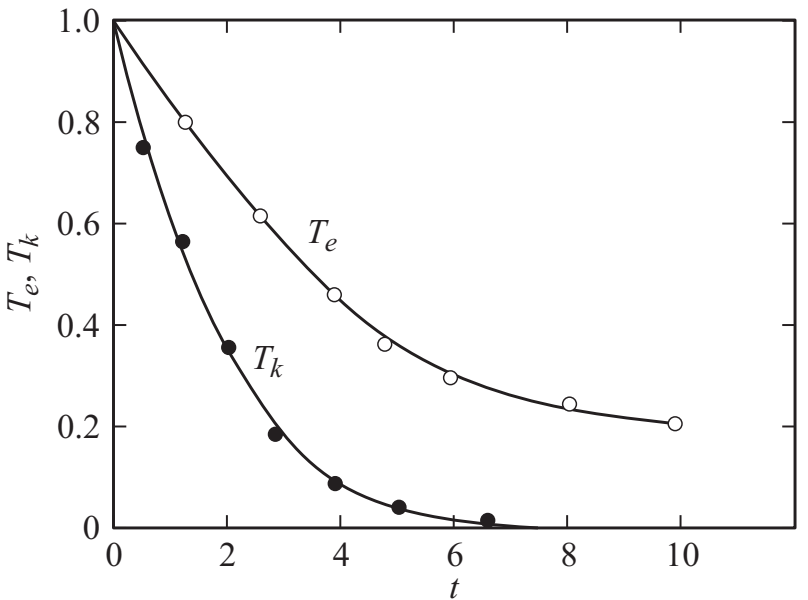

Рис. 1. Электронная $T_{e}$ и ионная $T_{i}$ температуры при разлете лазерной плазмы. $n=10^{19} \mathrm{~cm}^{-3}, R_{0}=3 \cdot 10^{-2} \mathrm{~cm}, T_{e}=$ $=T_{i}=10 \mathrm{eV}$.
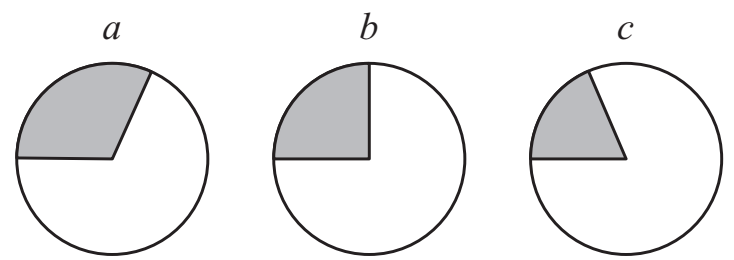

$\mathrm{Al}, \mathrm{Ti}, \mathrm{V}, \mathrm{Nd}, \mathrm{Pb}, \mathrm{Ga}$

$\mathrm{Ar}, \mathrm{J}, \mathrm{Cu}, \mathrm{Be}, \mathrm{Pt}, \mathrm{Au}, \mathrm{Zn}$

$\mathrm{Mo}, \mathrm{Fe}, \mathrm{In}, \mathrm{Pb}, \mathrm{Lu}, \mathrm{Nb}, \mathrm{W}$

Рис. 2. Номограммы энергий элементов с близкими потенциалами ионизаций: $a-\varphi_{a}+\varphi_{i}=20-30 \mathrm{eV} ; b-20-25 \mathrm{eV}$; $c-20-22 \mathrm{eV}$.

скорость движения границы сгустка стремится к некоторой постоянной величине. Конечная скорость разлета в рассматриваемом случае составляла $v_{k} \approx 1 \cdot 10^{6} \mathrm{~cm} / \mathrm{s}$, что соответствует кинетической энергии ионов железа $E_{k}=\frac{m v_{k}^{2}}{2}=25 \mathrm{eV}$. Эта величина, по-видимому, представляет собой нижнюю границу энергетического разброса ионов при заданных исходных параметрах плазмы. Отметим, что в рассматриваемом случае начальная удельная энергия лазерного импульса $W_{0}$ (приходящая на одну частицу) составляла около $120 \mathrm{eV}$. Пока плазменный сгусток не успевает рекомбинировать, значительная часть вложенной энергии сохраняется в виде потенциальной энергии. В процессе разлета плазмы ионы и электроны рекомбинируют и скорость рекомбинации со временем уменьшается из-за резкого падения плотности заряженных частиц.

Таким образом, ионы плазмы в конце некоторого времени $t$ обладают определенным зарядом и кинетической энергией. Другими словами, энергия лазерного излучения (искрового разряда или первичных ионов при ВИЭ), приходящаяся на одну частицу (в нашем случае $\left.W_{0} \approx 100 \mathrm{eV}\right)$, затрачивается как на диссоциацию и ионизацию атомов образца, так и в кинетическую энергию ионов $W_{k}\left(W_{0}=W_{k}+\varphi_{a}+\varphi_{i}\right)$, где $\varphi_{a}$ и $\varphi_{i}-$ 
энергии диссоциации и ионизации элементов соответственно. При этом чем больше $\left(\varphi_{a}+\varphi_{i}\right)$, тем меньше энергии ионов $W_{k}$. На рис. 2 представлены три группы элементов с энергиями, близкими по сумме энергий диссоциации $\varphi_{a}$ и однократной ионизации $\varphi_{i}\left(\varphi_{a}+\varphi_{i}\right)$. При этом площадь всего круга соответствует энергии, полученной атомом каждого элемента $\left(W_{0}\right)$, заштрихованная часть - потере энергии атомами $\left(\varphi_{a}+\varphi_{i}\right)$, а не штрихованная - количеству остаточной энергии атомов элементов этой группы. Из рисунка видно, что ионы первой группы $(a)$ в конце разлета обладают энергией $0.8 W_{0}$, второй $(b)-0.75 W_{0}$, третьей $(c)-$ менее чем $0.6 W_{0}$. Это означает, что на выходе из источника ионы обладают более чем 30\%-ным энергетическим разбросом, который и приводит к дискриминации их в ионно-оптическом тракте и анализаторе. Это особенно проявляется в тех масс-анализаторах, в которых энергия ионов играет решающую роль (времяпролетный, радиочастотный, квадрупольный и т.д.). Отсюда напрашивается, на наш взгляд, единственно верный вывод: для устранения дискриминаций, обусловленных диссоциацией, ионизацией и рекомбинацией, необходимо разделить эти процессы.

\section{Экспериментальная часть}

Эксперименты проводились как на аксиально-симметричном масс-спектрометре с лазерной ионизацией $\mathrm{c}$ интенсивностью излучения $q=10^{9} \mathrm{~W} / \mathrm{cm}^{2}$ и длительностью $\tau=10^{-8} \mathrm{~s}$, так и на ИСП-МС фирмы Agilent Technologies (США). Для расчета CRS интенсивности аналитических линий определяли по 25 одноэлементным образцам. Образцы для ИСП готовили в азотном или соляном растворах с одинаковыми концентрациями определяемых элементов - $10 \mu \mathrm{g} / \mathrm{l}$.

Настройку прибора проводили по раствору, содержащему по $10 \mu \mathrm{g} / 1$ иттрия, церия и талия в $1 \%$-ом азотнокислом растворе. Регистрировались линии однозарядных ионов. В качестве внутреннего стандарта выбрали $\mathrm{Lu}$ (лютеций), так как $\varphi_{\mathrm{Lu}}^{+}=5.4 \mathrm{eV}$ является примерно средней для определяемых элементов. Экспериментальные значение CRS-элементов определяли по формуле

$$
\mathrm{CRS}_{\mathrm{Lu}}^{x}=\frac{J_{x} n_{\mathrm{Lu}}}{J_{\mathrm{Lu}} n_{x}}
$$

где $n_{\mathrm{Lu}}$-содержание $\mathrm{Lu}$ в растворе, $n_{x}$ - содержание определенного элемента в растворе, $J_{x}$ и $J_{\mathrm{Lu}}-$ аналитические сигналы.

Эффективность ионизации атомов в ИСП рассчитывали по уравнению Саха-Эггерта

$$
\frac{a_{x}}{1-a_{x}}=\left(\frac{2 m_{e} k T}{n_{e} h^{3}}\right)^{3 / 2} \frac{2 Z_{x}^{+}}{Z_{x}^{0}} e^{-\varphi_{x}} / k T,
$$

где $a_{x}$ - степень ионизации атомов элемента $x, \varphi_{x}-$ потенциал ионизации, $Z_{x}^{+}$и $Z_{x}^{0}-$ суммы по состоянию иона и атома.

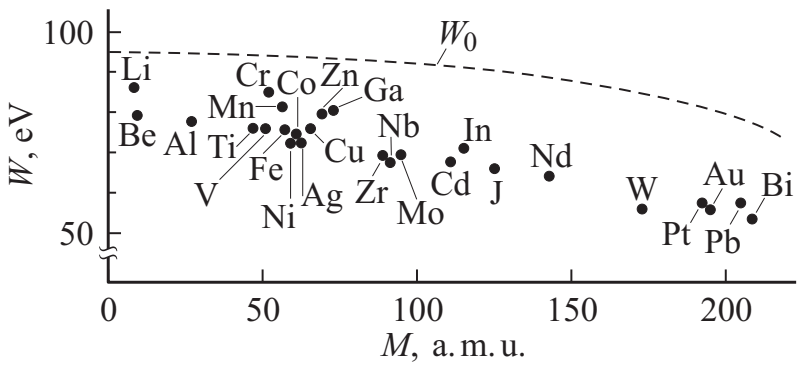

Рис. 3. Энергетический разброс ионов на выходе из источника ионов.

Отметим, что корреляция между экспериментально определенным CRS и энергией атомизации в наших экспериментах не была обнаружена. Коэффициент корреляции составил всего 0.03. Это означает, что в ИСП отсутствует селективность испарения, которая может приводить к различиям элементного состава газовой фазы и исходного раствора. Это позволяет рассчитать CRS по формуле

$$
K_{\mathrm{IS}}^{x}=\exp \left(\frac{\varphi_{x}-\varphi_{\mathrm{IS}}}{k T_{u}}\right) F\left(\frac{M_{x}}{M_{\mathrm{IS}}}\right),
$$

где $T_{u}$ - температура ионизации и $\varphi_{x}, \varphi_{\mathrm{IS}}-$ энергии однократных ионизаций искомого элемента и внутреннего стандарта, $M_{x}$ и $M_{\text {IS }}$ - массы соответствующих элементов, $F$ - приборный фактор, учитывающий зависимость чувствительности от массы однозарядных ионов.

На рис. 3 представлена зависимость измеренных энергий 24-х одноэлементных образцов на выходе из масс-анализатора. Как видно из рисунка, ряд элементов (например, $\mathrm{Li}, \mathrm{Be}, \mathrm{Cr}, \mathrm{Mn}, \mathrm{Zn}, \mathrm{Ga}$ ) приобрели дополнительные энергии, как нам кажется, за счет рекомбинации двух и более зарядных ионов, тогда как элементы W, $\mathrm{Pt}, \mathrm{Au}, \mathrm{Pb}, \mathrm{Bi}, \mathrm{Nd}$ и другие содержали в основном однократно заряженные ионы и поэтому сохранили свои энергии.

Как видно из таблицы, CRS рассчитанный по результатам исследований на ИСП-МС, намного ближе к единице, чем при других методах ионизации (в частности, лазерной). Это объясняется тем, что исследуемое вещество, растворенное в кислоте вместе с подающимся аргоном, в виде аэрозоля впрыскивается в плазму через центральный канал плазмы, где диссоциируется на атомы, которые затем ионизируются.

Другими словами, в ИСП-МС процессы диссоциации и ионизации практически отделены друг от друга, поэтому ионы, извлекаемые из индуктивной связанной плазмы энергетически менее разбросаны, и значит меньше отличаются по CRS, чем в лазерной (искровой ВИЭ) масс-спектрометрии.

Другим способом разделения процессов диссоциации и ионизации в МСП-МС является лазерная абляция, заключающаяся в испарении элементов вещества с поверхности образца лазерным импульсом низкой мощности. Получаемый в результате такого взаимодействия 
Коэффициенты относительной чувствительности разных элементов, рассчитанных по результатам экспериментов лазерной $\mathrm{MC}\left(\mathrm{CRS}_{l}\right)$ и индуктивно-связанной плазмы MC $\left(\mathrm{CRS}_{I}\right)$

\begin{tabular}{c|c|c|c|c|c|c|c|c|c}
\hline Элементы & $\varphi_{x}^{0} \mathrm{eV}[19,20]$ & $\varphi_{x}^{+} \mathrm{eV}[21]$ & $\mathrm{CRS}_{l}$ & $\mathrm{CRS}_{I}$ & Элементы & $\varphi_{x}^{0} \mathrm{eV}[19,20]$ & $\varphi_{x}^{0} \mathrm{eV}^{2}[21]$ & $\mathrm{CRS}_{l}$ & $\mathrm{CRS}_{I}$ \\
\hline $\mathrm{W}$ & 14.20 & 7.98 & 69.672 & 4.332 & $\mathrm{~V}$ & 8.47 & 6.74 & 127.871 & 4.361 \\
$\mathrm{Nb}$ & 11.94 & 6.77 & 79.763 & 6.915 & $\mathrm{Cd}$ & 7.82 & 8.99 & 89.236 & 7.015 \\
$\mathrm{Ni}$ & 11.24 & 7.63 & 95.317 & 5.449 & $\mathrm{Be}$ & 7.64 & 9.32 & 65.331 & 3.951 \\
$\mathrm{Bi}$ & 10.92 & 8.32 & 101.347 & 6.321 & $\mathrm{Li}$ & 7.63 & 5.39 & 84.423 & 5.771 \\
$\mathrm{Mo}$ & 10.86 & 7.18 & 98.312 & 5.215 & $\mathrm{Co}$ & 7.62 & 7.86 & 67.147 & 5.917 \\
$\mathrm{Pt}$ & 9.73 & 8.96 & 111.358 & 4.517 & $\mathrm{Ag}$ & 7.60 & 7.57 & 91.746 & 6.911 \\
$\mathrm{Au}$ & 9.69 & 9.22 & 80.126 & 5.812 & $\mathrm{Zn}$ & 7.25 & 9.39 & 108.265 & 5.441 \\
$\mathrm{Nd}$ & 9.37 & 6.32 & 81.147 & 6.217 & $\mathrm{Ga}$ & 6.94 & 6.00 & 74.376 & 4.217 \\
$\mathrm{Zr}$ & 9.18 & 6.93 & 100.340 & 6.410 & $\mathrm{In}$ & 6.82 & 5.78 & 70.123 & 5.03 \\
$\mathrm{~Pb}$ & 8.84 & 7.41 & 125.340 & 5.331 & $\mathrm{Cr}$ & 6.56 & 5.76 & 78.355 & 4.321 \\
$\mathrm{Al}$ & 8.83 & 5.89 & 102.321 & 7.362 & $\mathrm{~J}$ & 6.43 & 10.44 & 79.031 & 5.621 \\
$\mathrm{Ti}$ & 8.73 & 6.83 & 115.012 & 6.223 & $\mathrm{Fe}$ & 6.35 & 7.89 & 84.215 & 4.561 \\
$\mathrm{Cu}$ & 8.51 & 7.72 & 70.813 & 4.147 & $\mathrm{Mn}$ & 6.12 & 7.34 & 72.383 & 6.553
\end{tabular}

газообразный и мелкодисперсный аэрозоль потоком аргона транспортируется в осевую зону факела индуктивно связанной плазмы. Дальнейшие процессы аналогичны варианту ввода в плазму аэрозоля растворов.

Количественные масс-спектрометрические исследования состава ряда образцов чистых металлов $(\mathrm{Fe}, \mathrm{Cu}$, $\mathrm{Al}$, Ni и мн. др.) в аксиально-симметричном массанализаторе с помощью лазерной плазмы и ИСП показали неадекватность результатов. Расчет CRS для первого случая составлял от 120 до 70, а для второго от 4 до 7 (см. таблицу).

Что касается дискриминации ионов, обусловленных энергетическим разбросом при их детектировании с помощью вторично-электронного умножителя, то здесь можно выделить два эффекта. Первый - зависимость коэффициента усиления ВЭУ от массы ионов, второй от их энергии [18]. В большинстве случаев эта зависимость носит нелинейный характер, что приводит к значительной потере информации о частицах с малой энергией, т.е. малоинтенсивные частицы (частицы с меньшей концентрацией), но с большей энергией на выходе детектора дают аналитический сигнал больший, чем высокоинтенсивные частицы с малой энергией.

Это особенно ощущается во времяпролетных массанализаторах с аксиально-симметричным электрическим полем. Для устранения такой дискриминации нами разработана электрическая схема питания масс анализатора и ВЭУ, которая компенсирует разность энергий ионов с различными массами при пролете их между выходной сеткой, установленной на выходе анализатора, и детектором [22], и на ВЭУ поступают ионы с одинаковой энергией.

\section{Заключение}

Известно, что масс-спектрометрический анализ твердых тел представляет собой совокупность следующих этапов. Диссоциация атомов пробы, ионизация, рекомбинация ионов при разлете плазмы в вакуум, извлечение и их регистрация. В настоящей работе показано, что все эти этапы играют роль в формировании CRS. Если после первых двух этапов ионы всех элементов приобретают достаточный энергетический разброс, что приводит к различию CRS различных элементов, то рекомбинация „нивелирует“ различие между ионами. Другими словами, отношение числа однозарядных ионов разных элементов в масс-спектре приближается к отношению числа частиц этих же элементов в первоначальном плазменном сгустке.

Энергетический разброс, приобретенный ионами различных элементов, обусловлен разницей энергий диссоциации и потенциалов ионизации этих элементов, несмотря на то, что атомы всех элементов получают одинаковую энергию при лазерном излучении (в нашем случае более $100 \mathrm{eV}$ ).

Эксперименты показали, что для реализации безэталонного анализа твердых тел (т.е. для уменьшения CRS различных элементов) целесообразно разделить процессы диссоциации и ионизации по времени и (или) по пространству. Такая методика осуществлена в массспектрометрах с индуктивно-связанной плазмой (ИСП$\mathrm{MC})$.

При масс-спектрометрах с источниками ионизации высокотемпературной плазмой (лазерной, искровой и ВИЭ) для уменьшения дискриминации, связанной с энергетическим разбросом ионов, достаточно выравнивать их энергии перед детектированием.

Авторы выражают благодарность ведущему сотруднику к.т.н. Гасановой Сабине И. за активное участие в обсуждении и оформлении настоящей статьи

\section{Список литературы}

[1] Ганеев А.А., Губаль А.Р., Потапов С.Б., Тюкальцев Р.В. // Журн. аналит. хим. 2009. Т. 64. № 7. С. 715-728.

[2] Рамендик Г.И., Фатюшина Е.В., Степанов А.И. // Журн. аналит. хим. 2001. Т. 56. № 6. С. 561-573. 
[3] Рамендик Г.И., Манзон Б.М., Тюрин Д.А. // Журн. аналит. хим. 1989. Т. 44. № 6. С. 996-1007.

[4] Соколов А.В. Научная сессия МИФИ, 2005. Т. 9. С. 41.

[5] Гашимов А.М., Нуриев К.З., Гурбанов К.Б., Нурубейли З.К., Нурубейли Т.К. // ЖТФ. 2009. Т. 79. Вып. 8. С. 99 103.

[6] Тюрин Д.А., Рамендик Г.И., Черноглазова Г.И. // Журн. аналит. хим. 1989. Т. 44. № 12. С. 2157-2164.

[7] Малахов Ю.Б., Астахова С.А., Муштакова С.П. // Журн. аналит. хим. 2009. Т. 64. № 5. С. 495-503.

[8] Рублинская Ю.Б., Ильиных Е.О., Савушкин В.В. // Журн. аналит. хим. 2009. Т. 64. № 5. С. 525-528.

[9] Салман Манучар, Нурубейли 3.К., Нуриев К.3., Нурубейли T.К. // Электрон. обработка матер. 2011. Т. 47. № 3. C. 85-89.

[10] Гашимов А.М., Нуриев К.З., Салман Манучар и др. // Электрон. обработка матер. 2008. № 2. С. 98-104.

[11] Быковский Ю.А., Неволин В.Н. Лазерная масс-спектрометрия. М.: Энергоатомиздат, 1985. $128 \mathrm{c.}$

[12] Арефьев И.М., Беляев Н.Е., Комлева А.А. // Журн. аналит. хим. 2006. Т. 61. № 1. С. 50-54.

[13] Афанасьев Ю.В., Басов Н.Г. Взаимодействие мощного лазерного излучения с плазмой. М.: Радиотехника, 1978. T. $17.246 \mathrm{c}$.

[14] Рамендик Г.И. Элементный масс-спектрометрический анализ твердых тел. М.: Химик, 1993. 192 с.

[15] Быковский Ю.А., Сильнов С.М. Рекомбинация ионов лазерной плазмы. М.: Пр. МИФИ, 1987. № 008-87. 24 с.

[16] Васюта Ю.Б., Гречишников А.Б., Держиев В.И. Тр. VII Междунар. симп. по электроизоляции и электрическим разрядам в вакууме. Новосибирск. 1996.

[17] Нурубейли З.К., Нуриев К.З., Гурбанов К.Б. и др. // Приборы и техника эксперимента. 2014. № 3. С. 84-86.

[18] Айндбунд Н.Р., Поляков Б.В. Вторичные электронные умножители. М.: Энергоиздат, 1981.

[19] Эмсли Джс. Элементы / Пер. с англ. 1993. М.: Мир, 117 с. Справочник химика. Электронный ресурс. Режим доступа: 21/http//chem21.info/757385//

[20] Краснов К.С. Молекулы и химическая связь. М.: Высшая школа, 1984. $295 \mathrm{c.}$

[21] Чупахин М.С., Крючкова О.И., Рамендик Г.И. Аналитические возможности искровой масс-спектрометрии. М.: Атомиздат, 1972. $224 \mathrm{c}$.

[22] Нурубейли З.К., Нуриев К.З., Гурбанов К.Б., Нурубейли T.К. // Электрон. обработка матер. 2016. № 2. С. 127 131. 